\title{
Cluster features of stable and unstable nuclei in the $p$-shell region
}

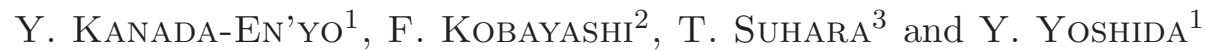

${ }^{1}$ Department of Physics, Kyoto University, Kyoto 606-8502, Japan

${ }^{2}$ Department of Physics, Niigata University, Niigata 950-2181, Japan

${ }^{3}$ Matsue College of Technology, Matsue 690-8518, Japan

\begin{abstract}
Cluster aspects in light unstable nuclei as well as light stable nuclei are discussed. We focus on cluster structures of excited states in ${ }^{12} \mathrm{C}$, ${ }^{9} \mathrm{Li}$, and ${ }^{10} \mathrm{Be}$. The rotation of $3 \alpha$ cluster gas states in ${ }^{12} \mathrm{C}$ and the ${ }^{6} \mathrm{He}+t$ cluster resonances in ${ }^{9} \mathrm{Li}$ are theoretically studied.
\end{abstract}

\section{Introduction}

In the recent experimental and theoretical studies, it has been revealed that a variety of cluster structures appear in unstable nuclei as well as stable nuclei (for instance, Refs. [1-4] and references therein). This fact indicates that cluster is one of the essential features in nuclei. Needless to say, the mean-field feature is another essential feature. The coexistence of cluster and mean-field features brings rich phenomena in nuclear systems.

${ }^{12} \mathrm{C}$ is a typical example where cluster and mean-field features coexist. The ground state of ${ }^{12} \mathrm{C}$ is a mean-field state dominated by the $p_{3 / 2}$-shell closed configuration. With energy around $100 \mathrm{MeV}$, all twelve nucleons of ${ }^{12} \mathrm{C}$ can break up, and the system evolves to a nucleon gas state. In the energy region around $10 \mathrm{MeV}$, much below the nucleon gas state, three $\alpha$ clusters develop in excited states of ${ }^{12} \mathrm{C}$. The energy for the $3 \alpha$ cluster excitation is much smaller than the energy for the nucleon gas state, implying that the mean-field and cluster states coexist in low-energy spectra of ${ }^{12} \mathrm{C}$.

This is an Open Access article distributed under the terms of the Creative Commons Attribution License 4.0, which permits unrestricted use, distribution, and reproduction in any medium, provided the original work is properly cited 


\section{EPJ Web of Conferences}

Moreover, the cluster feature contributes to the ground state structure of ${ }^{12} \mathrm{C}$. Because of many-body correlation, $\alpha$ clusters are formed at the nuclear surface even in the ground state, where clusters are largely overlapping with each other to form a compact triangle state of the $3 \alpha$ structure. This cluster feature is different from the spatially developed $3 \alpha$ cluster structures in excited states and it is regarded as a kind of ground state correlation. Namely, the ground state of ${ }^{12} \mathrm{C}$ is the $p_{3 / 2}$-shell closed configuration with the mixing of the $3 \alpha$ cluster component. From the standpoint of the cluster structure, the ground state is regarded as the compact $3 \alpha$ state containing the cluster breaking component.

Historically, many cluster structures have been found mainly in light stable nuclei. In these years, various cluster structures have been suggested in heavier-mass nuclei in $s d$-shell and $p f$-shell regions. For instance, in ${ }^{32} \mathrm{~S}$, $\alpha+{ }^{28} \mathrm{Si}$ cluster states have been suggested recently $[5,6]$. In ${ }^{32} \mathrm{~S}$, also the superdeformation with the ${ }^{16} \mathrm{O}+{ }^{16} \mathrm{O}$ cluster structure has been predicted in the comparable energy region in the theoretical study, and the ${ }^{16} \mathrm{O}+{ }^{16} \mathrm{O}$ molecular resonances have been known in much higher energy region $[1,3,4$, 7]. The possible coexistence of the $\alpha+{ }^{28} \mathrm{Si}$ cluster states and the ${ }^{16} \mathrm{O}+{ }^{16} \mathrm{O}$ cluster states is one of the interesting issues to be solved.

Further rich cluster phenomena are expected in unstable nuclei owing to existence of excess nucleons. As predicted in Ikeda's threshold rule [8], remarkable cluster structures with spatial development have been suggested in excited states near the threshold energy. In neutron-rich nuclei, many new cluster states containing exotic clusters have been suggested in theoretical and experimental studies: $\mathrm{He}+\mathrm{He}$ cluster states in Be isotopes, ${ }^{10} \mathrm{Be}+\alpha$ states in ${ }^{14} \mathrm{C},{ }^{14} \mathrm{C}+\alpha$ states in ${ }^{18} \mathrm{O}$ and their mirror states, ${ }^{18} \mathrm{O}+\alpha$ states in ${ }^{22} \mathrm{Ne},{ }^{9} \mathrm{Li}+{ }^{6} \mathrm{He}$ states in ${ }^{15} \mathrm{~B},{ }^{6} \mathrm{He}+t$ states in ${ }^{9} \mathrm{Li}$, and so on.

Conventional cluster models based on the assumption of specific clusters such as $\alpha$ and ${ }^{16} \mathrm{O}$ are no longer applicable for new cluster states having exotic clusters as $t,{ }^{6} \mathrm{He},{ }^{8} \mathrm{He},{ }^{10} \mathrm{Be},{ }^{14} \mathrm{C}$, and ${ }^{18} \mathrm{O}$ in unstable nuclei. Since 1990's, many extended frameworks such as antisymmetrized molecular dynamics (AMD) $[3,9,10]$ and fermionic molecular dynamics (FMD) methods [11-13], and extended cluster models of the stochastic variational method [14,15], the GCM method [16,17], and the generalized two-center cluster model $[18,19]$ have been developed and applied for cluster study of unstable nuclei.

Our aim is to theoretically investigate various cluster phenomena to achieve a systematic understanding of nuclear systems. In this paper, we discuss cluster phenomena in $p$-shell nuclei based on the theoretical calculations mainly using the antisymmetrized molecular dynamics (AMD) method [3,9]. 
One the advantages of the AMD method is that the model can describe features of both cluster and mean field aspects in general nuclei. In particular, shell-model structures and cluster formation in low-lying states as well as developed cluster structures in excited states can be described within the AMD framework without assuming the existence of any clusters.

This paper is organized as follows. In the following section, the formulation of the AMD model is explained. Cluster structures of ${ }^{12} \mathrm{C}$ are studied based on the AMD+VAP calculation in section 3 , and ${ }^{6} \mathrm{He}+t$ cluster resonances in ${ }^{9} \mathrm{Li}$ are discussed based on the cluster GCM calculation in section 4. Finally a summery is given in section 5 .

\section{Formulation}

In the framework of antisymmetrized molecular dynamics (AMD) [3, 9], an $A$-nucleon wave function is given by a Slater determinant of Gaussian wave packets:

$$
\Phi_{\mathrm{AMD}}(\mathbf{Z})=\frac{1}{\sqrt{A !}} \mathcal{A}\left\{\varphi_{1}, \varphi_{2}, \ldots, \varphi_{A}\right\},
$$

where the $i$ th single-particle wave function is written as

$$
\begin{aligned}
\varphi_{i} & =\phi_{\mathbf{X}_{i}} \chi_{i} \tau_{i} \\
\phi_{\mathbf{X}_{i}}\left(\mathbf{r}_{j}\right) & \propto \exp \left\{-\nu\left(\mathbf{r}_{j}-\frac{\mathbf{X}_{i}}{\sqrt{\nu}}\right)^{2}\right\} \\
\chi_{i} & =\left(\frac{1}{2}+\xi_{i}\right) \chi_{\uparrow}+\left(\frac{1}{2}-\xi_{i}\right) \chi_{\downarrow} .
\end{aligned}
$$

Here $\phi \mathbf{X}_{i}$ and $\chi_{i}$ are spatial and spin functions, and $\tau_{i}$ is the isospin function which is fixed to be up (proton) or down (neutron). The width parameter $\nu$ is fixed for each nucleus. Accordingly, an AMD wave function is specified by a set of variational parameters, $\mathbf{Z} \equiv\left\{\mathbf{X}_{1}, \mathbf{X}_{2}, \cdots, \mathbf{X}_{A}, \xi_{1}, \xi_{2}, \cdots, \xi_{A}\right\}$, which indicate the Gaussian center positions and the spin orientations for all nucleons. These parameters are determined by the energy variation.

The AMD wave function is similar to the FMD wave function $[11,12]$. The major difference between the AMD and the FMD calculations is the effective interaction. Phenomenological effective interactions are usually used in the AMD calculation, while the effective interaction derived from the realistic interaction is used in the FMD calculation.

To study nuclear structure, energy variation and spin-parity projection are applied to the AMD wave functions. For the study of Be isotopes, the 


\section{EPJ Web of Conferences}

variation after spin-parity projection was performed in the AMD framework (VAP). For more details of the AMD method, the reader is referred to Ref. [3].

To study the cluster resonances of ${ }^{9} \mathrm{Li}$, the ${ }^{6} \mathrm{He}+t$-cluster GCM calculation is performed instead of the AMD method.

The effective nuclear interactions used in the present calculations are the Volkov force and the MV1 force [20,21] with a spin-orbit force supplemented by the Coulomb force.

\section{Cluster gas and rotation}

In ${ }^{12} \mathrm{C}$, we see a variety of cluster structures comprising $3 \alpha$ clusters. The ground state of ${ }^{12} \mathrm{C}$ is the compact triangle state of the $3 \alpha$ cluster structure with a mixing of the $p_{3 / 2}$-shell closed configuration, while $3 \alpha$ cluster structures develop in excited states especially near or above the $3 \alpha$ threshold energy.

In this decade, the idea of the $\alpha$ cluster gas state has been proposed by Tohsaki et al. $[22,23]$. In the $\alpha$ cluster gas state, $\alpha$ clusters are weakly interacting in low density like a gas. Because of the bosonic behavior of $\alpha$ particles in such a dilute system, this state is often discussed in relation with $\alpha$ condensation in a dilute nuclear matter, though the concept of condensation should not be directly applied to such a small system of only three $\alpha$ s.

In the result of the AMD calculation, where the $\alpha$ cluster formation and breaking are microscopically taken into account, many developed $3 \alpha$ cluster states are obtained in excited states near and above the $3 \alpha$ threshold energy, even though any clusters are not a priori assumed in the model. We obtained the $0_{2}^{+}$state with the feature of the $\alpha$ cluster gas, and also the $0_{3}^{+}$ state having the remarkable $3 \alpha$ cluster structure with an open triangle $3 \alpha$ configuration (the bending chain like structure).

It is interesting to study the rotation of these cluster states. Recently, new excited states in ${ }^{12} \mathrm{C}$ were experimentally observed. The $2_{2}^{+}$state at 9.84(0.06) $\mathrm{MeV}$ and the $4_{1}^{+}$state at $13.3 \mathrm{MeV}$ were reported by Itoh et al. [24] and Freer et al. [25, 26]. These excited states are candidates of band members starting from the cluster gas states, ${ }^{12} \mathrm{C}\left(0_{2}^{+}\right)$. In Fig. 1, the excitation energies of these $3 \alpha$ cluster states around are shown as well as the $0_{2,3,4}^{+}$states. The energy spectra of the $0_{2}^{+}, 2_{2}^{+}$, and $4^{+}$states show a rotational band feature, the linear dependence on $J(J+1)$.

However, it is questionable whether the cluster gas state constructs the 
usual rotational band of the rigid rotor. A more essential problem is "what is the rotation of the cluster gas state?". The cluster gas state has no intrinsic deformation. Naively, the spherical gas state has no degree of freedom for the quantum rotation because of the rotational invariance of the intrinsic state. Indeed, in the result of AMD, it is found that the band is not the rigid rotor rotation but the intrinsic structure change occurs in the band with the increase of the spin. When the spin goes up, the cluster gas state in the band-head ${ }^{12} \mathrm{C}\left(0_{2}^{+}\right)$changes into somewhat deformed state in the ${ }^{12} \mathrm{C}\left(2_{2}^{+}\right)$. Once the intrinsic system has the intrinsic deformation, it can rotate and construct a rotational band. This is one of the interpretations of the rotation of the cluster gas state.

The above-mentioned assignment of the $0_{2}^{+}, 2_{2}^{+}$, and $4^{+}$states is based on the rather strong $E 2$ transition of $2_{2}^{+} \rightarrow 0_{2}^{+}$obtained by the AMD calculation. However, the AMD result shows further strong E2 transition of $2_{2}^{+} \rightarrow 0_{3}^{+}$, which suggests the possible alternative band assignment that the $0_{3}^{+}$state can be the band-head state of the $2_{2}^{+}$state. The intrinsic structure of the $0_{3}^{+}$state is dominated by the open triangle $3 \alpha$ configuration with the large deformation similar to the $2_{2}^{+}$and $4^{+}$states. As a result of the large deformation, the remarkably strong $E 2$ transitions are obtained between these bands as shown in Fig. 1.

Another interesting problem is the stability of the linear chain $3 \alpha$ structure in $\mathrm{C}$ isotopes. According to AMD and FMD calculations $[10,13,27]$, it was found that the straight-line linear chain structure is not stable in ${ }^{12} \mathrm{C}$ but only the bending chain-like $3 \alpha$ structure with the open triangle configuration may appear in the $0_{3}^{+}$state a few $\mathrm{MeV}$ above the $0_{2}^{+}$state. The question is whether the $3 \alpha$ linear chain structure with excess neutrons can be stabilized in excited states of neutron-rich $\mathrm{C}$ such as ${ }^{14} \mathrm{C}$. The structure of ${ }^{14} \mathrm{C}$ was studied with the $\beta-\gamma$ AMD method [28]. As is expected, it was found that the $3 \alpha$ linear-chain structure is stabilized by additional neutrons in excited states of ${ }^{14} \mathrm{C}[29]$. Because of the elongated shape, the linear chain structure is predicted to construct a $K^{\pi}=0^{+}$rotational band with the small level spacing above the ${ }^{10} \mathrm{Be}+\alpha$ threshold energy.

\section{$4{ }^{6} \mathrm{He}+t$ and ${ }^{6} \mathrm{He}+{ }^{4} \mathrm{He}$ cluster states in ${ }^{9} \mathrm{Li}$ and ${ }^{10} \mathrm{Be}$}

The $K^{\pi}=0_{2}^{+}$band in ${ }^{10}$ Be has been extensively studied and its developed cluster structure has been discussed in experimental and theoretical works. The $0_{2}^{+}$state at $6.18 \mathrm{MeV}$ just below the ${ }^{6} \mathrm{He}+\alpha$ threshold energy is con- 

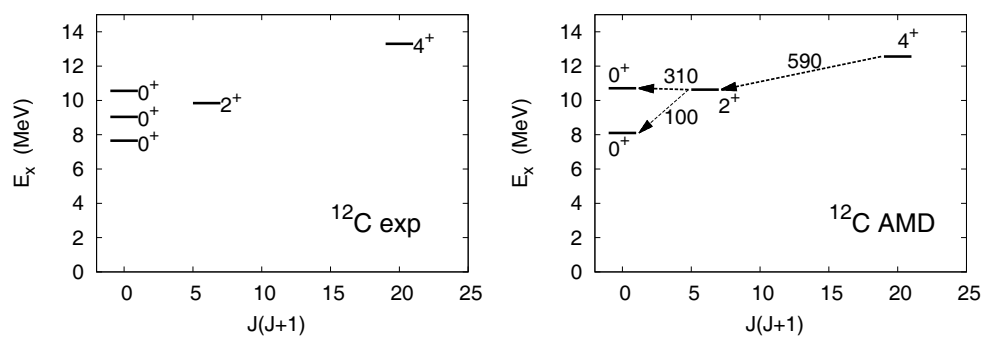

Figure 1: Energy levels of $3 \alpha$ cluster states of ${ }^{12} \mathrm{C}$. (Left) The experimental levels of $0^{+}, 2^{+}$, and $4^{+}$states for the $3 \alpha$ cluster states around $E_{x} \sim 10 \mathrm{MeV}$ which have been reported by recent experimental works by Itoh et al., [24] and Freer et al. $[25,26]$. The $0_{2}^{+}$state is also shown. (Right) The $0_{2}^{+}, 0_{3}^{+}, 2_{2}^{+}$, and $4_{2}^{+}$states obtained by the AMD+VAP calculation [27]. The calculated $B(E 2)$ values $\left(e^{2} \mathrm{fm}^{4}\right)$ are also shown by arrows.

sidered to be the band-head state of the $K^{\pi}=0_{2}^{+}$band. The $2^{+}$state at $7.54 \mathrm{MeV}$ and the $4^{+}$state at $10.2 \mathrm{MeV}$ have been experimentally suggested to have a developed ${ }^{6} \mathrm{He}+\alpha$-cluster structure [30-32] though the spin and parity of the $10.2 \mathrm{MeV}$ have not established yet [33]. These $2^{+}$and $4^{+}$states are considered to be the candidates for the members of the $K^{\pi}=0_{2}^{+}$band starting from the $0_{2}^{+}$state.

In the theoretical studies of ${ }^{10} \mathrm{Be}$, the ground and excited $K^{\pi}=0^{+}$bands have been described well having $2 \alpha+2 n$ cluster models. In the molecular orbital models [34-37], the $K^{\pi}=0_{2}^{+}$band is described by the $\sigma^{2}$ molecularorbital state where valence neutrons occupy the $\sigma$ orbital with two nodes along the $\alpha-\alpha$ direction. Sophisticated four-body calculations of $\alpha+\alpha+n+n$ have supported the $\sigma^{2}$ structure of the $K^{\pi}=0_{2}^{+}$band [15,17]. In Refs. [18, 19], the molecular orbital formation and the $\alpha+{ }^{6} \mathrm{He}$ asymptotic behaviors are described in a unified way, and the transition from the strong coupling ${ }^{6} \mathrm{He}+\alpha$ cluster state to the weak coupling one is investigated. It should be commented that the $2 \alpha$ cluster formation in neutron-rich Be isotopes has been indeed confirmed by the AMD calculations without assuming existence of $\alpha$ clusters [38,39].

The development of the ${ }^{6} \mathrm{He}+\alpha$ cluster structure in the excited states near the threshold energy is naively expected from the Ikeda's threshold rule which suggests appearances of developed cluster states near the corresponding threshold energy [8]. Similarly to the ${ }^{6} \mathrm{He}+\alpha$ cluster states in ${ }^{10} \mathrm{Be}$, one may expect the possible appearance of ${ }^{6} \mathrm{He}+t$ cluster states near the ${ }^{6} \mathrm{He}+t$ threshold energy in excited states of ${ }^{9} \mathrm{Li}$. It is a challenging issue 

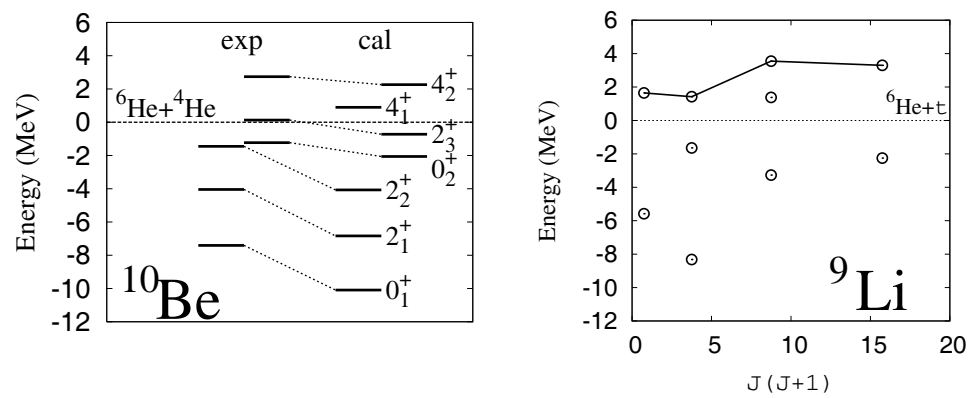

Figure 2: (Left) Energy levels of positive-parity states in ${ }^{10} \mathrm{Be}$. The calculated levels are obtained by the ${ }^{6} \mathrm{He}+{ }^{4} \mathrm{He}$-cluster GCM calculation. (Right) Energy levels of negative-parity states in ${ }^{9} \mathrm{Li}$ calculated by the ${ }^{6} \mathrm{He}+t$-cluster $\mathrm{GCM}$ method. The energies measured from the ${ }^{6} \mathrm{He}+{ }^{4} \mathrm{He}$ and ${ }^{6} \mathrm{He}+t$ threshold are plotted. The figures are taken from Ref. [40].

to search for such resonances of two neutron-rich clusters. In our previous study with the quadrupole deformation $(\beta-\gamma)$ constraint in a framework of AMD, we have shown an indication of a largely deformed state having a ${ }^{6} \mathrm{He}+t$ structure in the excited states of ${ }^{9} \mathrm{Li}[28]$.

To investigate the ${ }^{6} \mathrm{He}+t$-cluster states near the ${ }^{6} \mathrm{He}+t$ threshold in ${ }^{9} \mathrm{Li}$, we perform GCM calculations with a ${ }^{6} \mathrm{He}+t$ cluster model [40]. The calculated energy levels measured from the ${ }^{6} \mathrm{He}+t$ threshold energy are shown in Fig. 2. In the calculated result, we obtain ${ }^{6} \mathrm{He}+t$ cluster resonances a few $\mathrm{MeV}$ above the ${ }^{6} \mathrm{He}+t$ threshold energy. The cluster resonances $J^{\pi}=1 / 2_{2}^{-}$, $3 / 2_{3}^{-}, 5 / 2_{2}^{-}$and $7 / 2_{2}^{-}$form a $K^{\pi}=1 / 2^{-}$rotational band, and show strong in-band $E 2$ transitions. They show remarkably developed ${ }^{6} \mathrm{He}+t$ cluster structure.

Compared with the $K^{\pi}=0_{2}^{+}$band of ${ }^{10} \mathrm{Be}$, in which the ${ }^{6} \mathrm{He}$ cluster strongly couples to the neighboring ${ }^{4} \mathrm{He}$ cluster, the ${ }^{6} \mathrm{He}$ cluster in the ${ }^{6} \mathrm{He}+t$ cluster states of ${ }^{9} \mathrm{Li}$ couples more weakly to the $t$ cluster. It means that the ${ }^{6} \mathrm{He}+t$-cluster states have dominantly the ${ }^{6} \mathrm{He}\left(0^{+}\right)$component, that is, the ${ }^{6} \mathrm{He}$ cluster in the ${ }^{6} \mathrm{He}+t$ resonances is rather spherical. It is contrast to the deformed ${ }^{6} \mathrm{He}$ cluster in the $K^{\pi}=0_{2}^{+}$band of ${ }^{10} \mathrm{Be}$, in which the ${ }^{4} \mathrm{He}$ cluster is sitting on the head of a deformed ${ }^{6} \mathrm{He}$ cluster. The reason for the weaker coupling to the $t$ cluster than the coupling to ${ }^{4} \mathrm{He}$ is that valence neutrons feel the weaker potential from the $t$ cluster and therefore they do not move around the whole $\alpha+t$ core but tend to localize around the $\alpha$ core. This is different from the case of ${ }^{10} \mathrm{Be}$, in which neutrons are moving in the molecular $\sigma$-orbital around the $2 \alpha$-cluster core. 
There is no experimental information for the ${ }^{6} \mathrm{He}+t$ cluster resonances. It is a challenging problem to experimentally search for such cluster states consisting of exotic clusters, ${ }^{6} \mathrm{He}$ and $t$, which are unstable nuclei themselves.

\section{Summary}

Various kinds of cluster structures appear in nuclear systems depending on the neutron and proton numbers as well as on the excitation energy. We focused on cluster structures of $p$-shell nuclei. In particular, we discussed cluster features of excited states in ${ }^{12} \mathrm{C},{ }^{9} \mathrm{Li}$, and ${ }^{10} \mathrm{Be}$.

Cluster structures of ${ }^{12} \mathrm{C}$ were studied with the AMD+VAP calculation. In the energy region near and above the $3 \alpha$ threshold energy, many developed $3 \alpha$ cluster states are obtained in the theoretical result. The rotation of these $3 \alpha$ cluster states were discussed. Highly excited cluster states of ${ }^{9} \mathrm{Li}$ were studied with cluster GCM calculations. The present work suggests the ${ }^{6} \mathrm{He}+t$ cluster resonance states near the threshold energy in ${ }^{9} \mathrm{Li}$. These states are peculiar because they consist of exotic clusters that are themselves unstable nuclei. The analogy and difference between the clustering properties of ${ }^{9} \mathrm{Li}$ and ${ }^{10} \mathrm{Be}$ were also discussed.

\section{Acknowledgments}

The computational calculations of this work were performed by using the supercomputers at YITP. This work was supported by JSPS KAKENHI.

\section{References}

[1] S. Ohkubo et al., Prog. Theor. Phys. Suppl. 132, 1 (1998).

[2] W. von Oertzen, M. Freer and Y. Kanada-En'yo, Phys. Rep. 432, 43 (2006).

[3] Y. Kanada-En'yo and H. Horiuchi, Prog. Theor. Phys. Suppl. 142, 205 (2001); Y. Kanada-En'yo M. Kimura and H. Horiuchi, C. R. Physique 4, 497 (2003); Y. Kanada-En'yo, M. Kimura and A. Ono, PTEP 2012 01A202 (2012).

[4] H. Horiuchi, K. Ikeda, and K. Katō "Recent Developments in Nuclear Cluster Physics" Prog. Theor. Phys. Suppl. 192, 1 (2012). 
[5] T. Lönnroth, et al., Eur. Phys. J. A 46, 5 (2010); T. Lönnroth, et al., J. Phys. G 38, 035107 (2011).

[6] M. Itoh, S. Kishi, H. Sakaguchi, H. Akimune, M. Fujiwara, U. Garg, K. Hara and H. Hashimoto et al., arXiv:1310.0169 [nucl-ex].

[7] M. Kimura and H. Horiuchi, Phys. Rev. C 69, 051304 (2004).

[8] K. Ikeda, N. Tagikawa, and H. Horiuchi, Prog. Theor. Phys. Suppl. extra number, 464 (1968);

K. Ikeda et al., Prog. Theor. Phys. Suppl. 52, 1 (1972).

[9] Y. Kanada-En'yo, H. Horiuchi and A. Ono, Phys. Rev. C 52, 628 (1995);

Y. Kanada-En'yo and H. Horiuchi, Phys. Rev. C 52, 647 (1995).

[10] Y. Kanada-En'yo, Phys. Rev. Lett. 81, 5291 (1998).

[11] H. Feldmeier, K. Bieler and J. Schnack, Nucl. Phys. A 586, 493 (1995).

[12] T. Neff and H. Feldmeier, Nucl. Phys. A 713, 311 (2003).

[13] M. Chernykh, H. Feldmeier, T. Neff, P. von Neumann-Cosel and A. Richter, Phys. Rev. Lett. 98, 032501 (2007).

[14] K. Varga, Y. Suzuki and R. G. Lovas, Nucl. Phys. A 571, 447 (1994).

[15] K. Arai, Y. Ogawa, Y. Suzuki and K. Varga, Phys. Rev. C 54, 132 (1996); K. Arai, Phys. Rev. C 69, 014309 (2004).

[16] D. L. Hill and J. A. Wheeler, Phys. Rev. 89, 1102 (1953); J. J. Griffin and J. A. Wheeler, Phys. Rev. 108, 311 (1957).

[17] P. Descouvemont, Nucl. Phys. A 699, 463 (2002).

[18] M. Ito, K. Kato and K. Ikeda, Phys. Lett. B 588, 43 (2004).

[19] M. Ito, Phys. Lett. B 636, 293 (2006).

[20] A. B. Volkov, Nucl. Phys. 74, 33 (1965).

[21] T. Ando, K. Ikeda, and A. Tohsaki, Prog. Theory. Phys. 64, 1608 (1980).

[22] A. Tohsaki, H. Horiuchi, P. Schuck and G. Ropke, Phys. Rev. Lett. 87, 192501 (2001). 


\section{EPJ Web of Conferences}

[23] Y. Funaki, A. Tohsaki, H. Horiuchi, P. Schuck and G. Ropke, Phys. Rev. C 67, 051306 (2003).

[24] M. Itoh, H. Akimune, M. Fujiwara, U. Garg, N. Hashimoto, T. Kawabata, K. Kawase and S. Kishi et al., Phys. Rev. C 84, 054308 (2011).

[25] M. Freer, S. Almaraz-Calderon, A. Aprahamian, N. I. Ashwood, M. Barr, B. Bucher, P. Copp and M. Couder et al., Phys. Rev. C 83, 034314 (2011).

[26] M. Freer, M. Itoh, T. Kawabata, H. Fujita, H. Akimune, Z. Buthelezi, J. Carter and R. W. Fearick et al., Phys. Rev. C 86, 034320 (2012).

[27] Y. Kanada-En'yo, Prog. Theor. Phys. 117, 655 (2007) [Erratum-ibid. 121, 895 (2009)].

[28] T. Suhara and Y. Kanada-En'yo Y, Prog. Theor. Phys. 123, 303 (2010).

[29] T. Suhara and Y. Kanada-En'yo, Phys. Rev. C 82, 044301 (2010).

[30] N. Soić et al., Europhys. Lett. 34, 7 (1996).

[31] M. Milin et al., Nucl. Phys. A753, 263 (2005).

[32] M. Freer et al., Phys. Rev. Lett. 96, 042501 (2006).

[33] N. Curtis et al., Phys. Rev. C 70, 014305 (2004).

[34] M. Seya, M. Kohno, and S. Nagata, Prog. Theor. Phys. 65, 204 (1981).

[35] W. von Oertzen, Z. Phys. A 354, 37 (1996); 357, 355 (1997).

[36] W. von Oertzen, Nuovo Cimento 110, 895 (1997).

[37] N. Itagaki and S. Okabe, Phys. Rev. C 61, 044306 (2000); N. Itagaki, S. Okabe and K. Ikeda, Phys. Rev. C 62, 034301 (2000).

[38] A. Dote, H. Horiuchi and Y. Kanada-En'yo, Phys. Rev. C 56, 1844 (1997).

[39] Y. Kanada-En'yo, H. Horiuchi and A. Doté, Phys. Rev. C 60, 064304 (1999).

[40] Y. Kanada-En'yo and T. Suhara, Phys. Rev. C 85, 024303 (2012). 